\title{
EFFECTS OF NANOCAPSULES CONTAINING ALL-TRANS-RETINOIC ACID UNDER HEMOLYTIC AND COAGULATION ACTIVITY
}

\section{Sousa Filho WPS}

Graduating in Biomedicine from the Franciscan University; walter_paixao@hotmail. com; orcid.org/0000-0001-5962-4908

\section{Homrich SSH}

Master and PhD candidate for the Postgraduate Program in Nanoscience of the Franciscan University; shayscheffer@yahoo.com.br; orcid/org 0000-0001-8673-2699

\section{Copetti PM}

Master in Nanosciences by the Franciscan University; Graduated in Biomedicine from the Franciscan University; priscilaa_marquezan@hotmail.com; orcid.org/00000003-1140-6236

\section{Peres DS}

Graduated in Biomedicine with qualification in Clinical Pathology (Clinical Analysis) and Molecular Biology by the Franciscan University; diullespat@hotmail.com; orcid. org/0000-0001-8850-5991

\section{Souza DV}

Graduated in Biomedicine from the Franciscan University; diully_desouza@hotmail. com; orcid.org/0000-0002-6126-2501

\section{Riéffel RC}

Specialist in Exercise Physiology from Universidade Veiga de Almeida; Master's degree in the Graduate Program in Nanoscience by the Franciscan University; rorieffel@gmail. com; orcid.org/0000-0002-7169-5124 


\section{Machado AK}

PhD and MS in Pharmacology from the Federal University of Santa Maria; Professor in the Biomedicine and Medicine courses at the Franciscan University; alencarkolinski@ gmail; orcid.org/0000-0003-2003-8420

\section{Ourique AF}

PostDoc by the Graduate Program in Nanosciences of the Franciscan University; PhD in Pharmaceutical Nanotechnology, Federal University of Rio Grande do Sul; Adjunct Professor I at the Franciscan University; alineourique@gmail.com; orcid.org/00000003-0828-774X

\section{Sagrillo MR}

PhD in Pharmacology, Pharmacogenomics, Federal University of Santa Maria; Master in Biological Sciences, Genetics area, Federal University of São Paulo; Adjunct Professor I at the Franciscan University; Rua Andradas, 1614, Building 4, 97010-032, Santa Maria, Rio Grande do Sul, Brazil; sagrillorm18@gmail.com; orcid.org/0000-0001-5659-159X

Abstract: The chemotherapeutic all-trans retinoic acid (ATRA) used in the treatment of Acute Promyelocytic Leukemia has adverse effects on its oral administration, with which we incorporated a system of drugs, the nanocapsules, in order to have a possible improvement in solubility, photosensitivity, lower toxicity, generating pharmacological efficacy. The objective was to evaluate and compare the hemolytic and coagulation activity of the free drug ( $\mathrm{AL}$ ), nanoencapsulated (NA) and the white nanocapsules (NB) by analyzing the results of hemolysis, Prothrombin Time (PT) and Activated Partial Thromboplastin Time (APTT). We developed a prospective study of treatments at different concentrations of $0.25 ; 0.5 ; 1.0 ; 1.5 ; 2.0 ; 2.5 \mu \mathrm{g} / \mathrm{mL}$. For the first test, all concentrations showed hemolytic activity, but when compared to NA with ATRA it is observed that these carriers induced lower hemolytic toxicity. In the PT test the nanoparticles at the two lowest concentrations remained in the physiological range (12 - 15 seconds). For the APTT test the three lowest concentrations remained within the control (25 - 35 seconds). Thus, we believe there is a promising benefit of using these nanoparticles developed and no doubt further studies will be performed to confirm the responses obtained here.

Keywords: Coagulation cascade. Hemolysis. Leukemia. 


\section{INTRODUCTION}

Bleeding is a frequent and potentially lethal complication in cancer patients. It may be directly related to the disease, to the antineoplastic treatment or to factors not related to the neoplasia itself. ${ }^{1}$

The control of this alteration in the site of the endothelial lesion includes the action of subendothelium, endothelium, platelets and plasma proteins, a process which it is called hemostasis. ${ }^{1}$

One of the clotting disorders severely encountered in the clinical course of many pathologies, especially Acute Promyelocytic Leukemia (APL) is Disseminated Intravascular Coagulation (DIC). DIC is currently defined as an acquired syndrome, characterized by the diffuse activation of intravascular coagulation, leading to the formation and deposition of fibrin in the microvasculature. ${ }^{2}$

The clinical course of APL in recent years has been modified from a rapidly fatal acute leukemia to one of the more curable AML subtypes. This revolutionary progress in the prognosis of the disease is essentially associated with the remarkable advances in its treatment, more specifically with the introduction of new therapeutic agents that act directly in the molecular alteration, as is the case of all-trans retinoic acid derived from vitamin A (ATRA) and arsenic trioxide (ATO).

After following the administration, a rapid increase in leukocytes is observed, which is accompanied by Differentiation Syndrome ${ }^{3}$ On the other hand, when administered orally, its bioavailability is reduced, and this can be attributed to its low solubility in water. Moreover, this type of treatment, when prolonged, provides a progressive reduction of its plasma concentration, which can be attributed to its increased metabolism and increased haemolytic toxicity. ${ }^{4}$

To reduce this disadvantage and adverse effect of free ATRA, this work aimed to incorporate the ATRA into a different drug delivery system, the nanocapsules, which when combined with the drug can improve the solubility, photosensitivity, toxicity, chemical stability, bioavailability and/or pharmacological efficacy. ${ }^{5}$ In addition, the ATRA is a lipophilic 
drug that is very poorly soluble in water, and its incorporation into lipid nanocarriers is an interesting alternative to allow its intravenous administration to replace oral use. ${ }^{6}$

Therefore, we used the determination of hemoglobin release in red blood cells as an in vitro model, as well as the possible effects on blood coagulation to study the toxicity of ATRA, ATRA nanocapsuled and blank nanocapsules.

\section{MATERIALS AND METHODS}

This work consisted of two stages: the first one to produce and characterize the nanocapsule suspensions, and a prospective in vitro study was carried out in which red blood cells and plasma were used as an experimental model to investigate the potential hemolytic and coagulation effects of the chemotherapeutic ATRA and its nanoencapsulated form.

The discard samples were obtained from healthy donors from peripheral blood, from the Laboratory of Clinical Analysis (LEAC) of the Universidad Franciscana under the Ethics Committee of the institution and CAAE registry: 31211214.4.0000.5306.

The samples were collected by venipuncture using Vacutainer tubes (BD Diagnostics, Plymouth, UK) containing EDTA (ethylenediaminetetraacetic acid) for hemolysis and sodium citrate for coagulation.

The nanoparticles loaded with ATRA (NA) and the blank nanocapsules (NB) - drugfree in the structure - were produced according to the formulation described by Ourique et al.,6 with some adaptations. For the preparation of the nanocapsules, the interfacial deposition method of the polymer, described by Fessi, Puisieux, Devissaguet, Ammoury, and Benita, ${ }^{7}$ was used.

The ATRA content found for the drug loaded nanocapsules was $98 \%$ at a concentration of $0.5 \mu \mathrm{g} / \mathrm{mL}$. The treatment for both tests was performed with ATRA, nanocapsuled ATRA and blank nanocapsule, in the following concentrations of $0.25 ; 0.5$; $1.0 ; 1.5 ; 2.0 ; 2.5 \mu \mathrm{g} / \mathrm{mL}$. 


\subsection{HEMOLYTIC ACTIVITY}

The methodology followed according to Dobrovolskaia et al.8 Blood was added to 1x PBS at 1: 1 ratio and centrifuged 15 minutes at $1000 \mathrm{rpm}$, the supernatant was discarded, and this procedure was performed three times. Subsequently, in a new tube containing 1 $\mathrm{mL}$ of $1 x$ PBS, $400 \mu \mathrm{L}$ of already washed red blood cells and $80 \mu \mathrm{L}$ of treatment were added. As negative control cells were used $(400 \mu \mathrm{L})$ and PBS $1 \times(1 \mathrm{~mL})$ and as positive control cells $(400 \mu \mathrm{L})$, PBS $1 \times(1 \mathrm{~mL})$ and H2O2 $(80 \mu \mathrm{L})$. The tubes were incubated in a 37 ${ }^{\circ} \mathrm{C}$ at $5 \% \mathrm{CO} 2$ for 1 hour. It was then centrifuged for 5 minutes at $1000 \mathrm{rpm}$ and $100 \mu \mathrm{L}$ of the supernatant to a 96-well plate, which was read in ELISA reader at $405 \mathrm{~nm}$.

\subsection{COAGULATION TEST}

This assay was performed according to protocol Nanotechnology Characterization Laboratory (Frederick National Laboratory for Cancer Research Leidos Biomedical Research, Inc. Frederick, MD 21702). Whole blood was collected and centrifuged 10 minutes at $2500 \mathrm{rpm}$. Then, $225 \mu \mathrm{L}$ of plasma was separated into wells along with $25 \mu \mathrm{L}$ of treatments and incubated at $37{ }^{\circ} \mathrm{C}$ for 30 minutes. Two independent experiments were performed in duplicate with different donors. It was later performed in reading coagulometer Quick Timer II (Drake) properly calibrated, according to the manufacturer's recommendations for TP Hemostasis tests (Labtest - Lot: 4008) and TTPA Hemostasis (Labtest - Lot: 4006). We used as reference for the TTPA normal value the interval between 25 and 35 seconds, while the baseline values for TP used the interval between 12 and 15 seconds according to the authors. ${ }^{9,10}$

\subsection{STATISTICAL ANALYSIS}

The hemolysis assay was performed in triplicate. The results were expressed as percentage of positive control (100\%), mean \pm standard deviation (S.D.). The analyzes were performed by variance (ANOVA) of 1-way, followed by Dunnett's post hoc test. Values with $p$ $<0.05$ were considered statistically significant. Coagulation was performed in two independent 
experiments, these in duplicate with different donors. The results were expressed as mean \pm S.D. and compared to the corresponding physiological times of each test.

\section{RESULTS}

The suspensions of nanocapsules produced had smooth and milky, and the white tone exhibited NBs and NAs exhibited slight yellowish tint due to the presence of the drug, which in its free form has a yellow color.

The average $\mathrm{pH}$ of the formulations was 6.1 and 6.3 for the NB to NA. The results are in accordance with the results reported by Fachinetto, Ourique, Lubini, Tedesco, Silva, and Beck,5 indicating that the formulations of nanocapsules produced according to $\mathrm{pH}$ have stability.

Regarding the size, the suspensions in this study showed an average diameter of $209 \mathrm{~nm}$ to $218 \mathrm{~nm}$ for NB and NA.

The PDI for the NB was 0.195 and for the NA of 0.158.

Figure1 belowshows theresultsregarding thehemolyticactivity of thechemotherapeutic ATRA in its free form (ATRA), ATRA nanoencapsulated (NA) and blank nanocapsule (NB). 


\section{hemolytic activity}

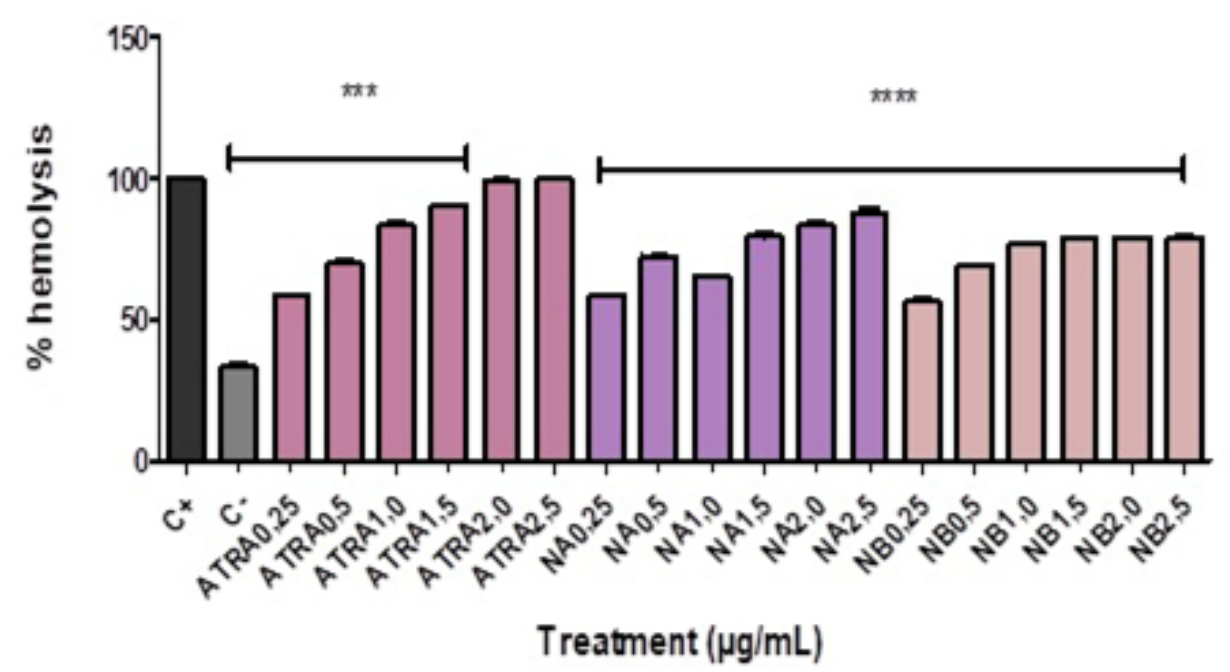

Figure 1 - Hemolysis assay with different treatments

Notes: Results expressed as percentage of positive control (100\%). Data were expressed as mean \pm standard deviation (SD). Analyzes were performed by 1-way ANOVA, followed by Dunnett's post hoc test. Values with $p<0.05$ were considered statistically significant.

The results found in the hemolytic activity of ATRA, evidenced the increase of the same, dependent concentration.

Performed a new $\mathrm{v}$ analysis and comparing free and nanocapsules concentrations for the presence of significant differences, as shown in Table 1

\begin{tabular}{llll}
\hline Concentration & ATRA & NA & “p” value \\
\hline $1,0 \mu \mathrm{g} / \mathrm{mL}$ & $83,49 \%$ & $65,1 \%$ & $<0,001$ \\
$1,5 \mu \mathrm{g} / \mathrm{mL}$ & $90 \%$ & $79 \%$ & $<0,001$ \\
$2,0 \mu \mathrm{g} / \mathrm{mL}$ & $100 \%$ & $83 \%$ & $<0,001$ \\
$2,5 \mu \mathrm{g} / \mathrm{mL}$ & $100 \%$ & $86 \%$ & $<0,001$ \\
\hline
\end{tabular}

Table 1 - Comparative analysis between treatments with $A L$ and NA through analysis of variance (ANOVA) of 1 way, followed by Tukey post hoc test

These results demonstrate that although the activity of the ATRA-loaded nanocapsules showed haemolytic activity, still these carriers induced lower haemolytic toxicity compared to ATRA. 
The prothrombin time (PT) and Activated Partial Thromboplastin Time (APTT) were measured by measuring the coagulant activity. As previously mentioned, blood from healthy patients, specifically isolated plasma, was used for this study. The results of coagulation tests are related to the type and concentration of treatments as can be observed in the figures below, being TP (Figure 2) and APTT (Figure 3). Where for the first test, only the treatments with the nanoparticles in the concentration of $1,0 \mu \mathrm{g} / \mathrm{mL}$ remained above the physiological range. In the second test, all are within the range. Results greater than $1.5 \mu \mathrm{g} / \mathrm{mL}$ are not represented graphically by producing an anticoagulant effect, values greater than 120 seconds and 240 seconds, respectively.

\section{Prothrombin Time}

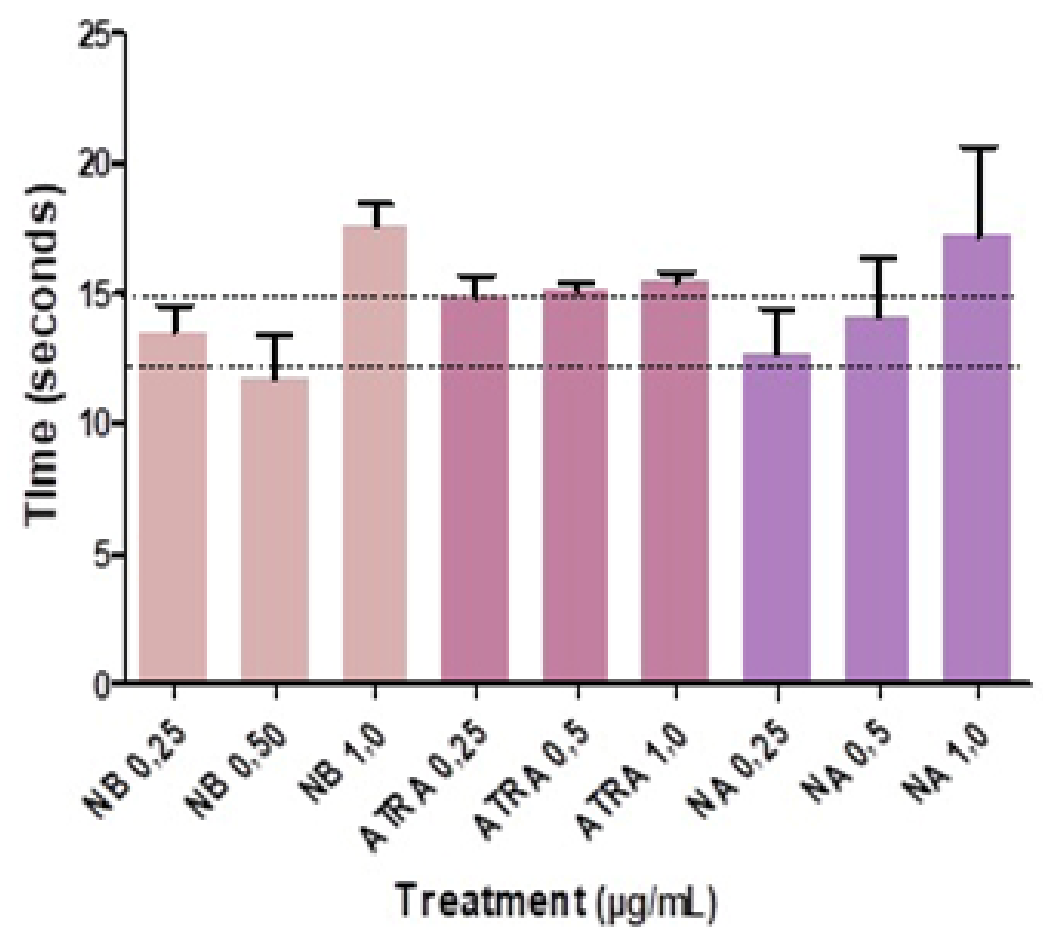

Figure 2 - Results of PT

Notes: The dotted boundaries correspond to expected physiological times for healthy donors (between 12 and 15 seconds). Mean \pm S.D. values from two independentexperiments and from different donors. 


\section{Activated Partial Thromboplastin Time}

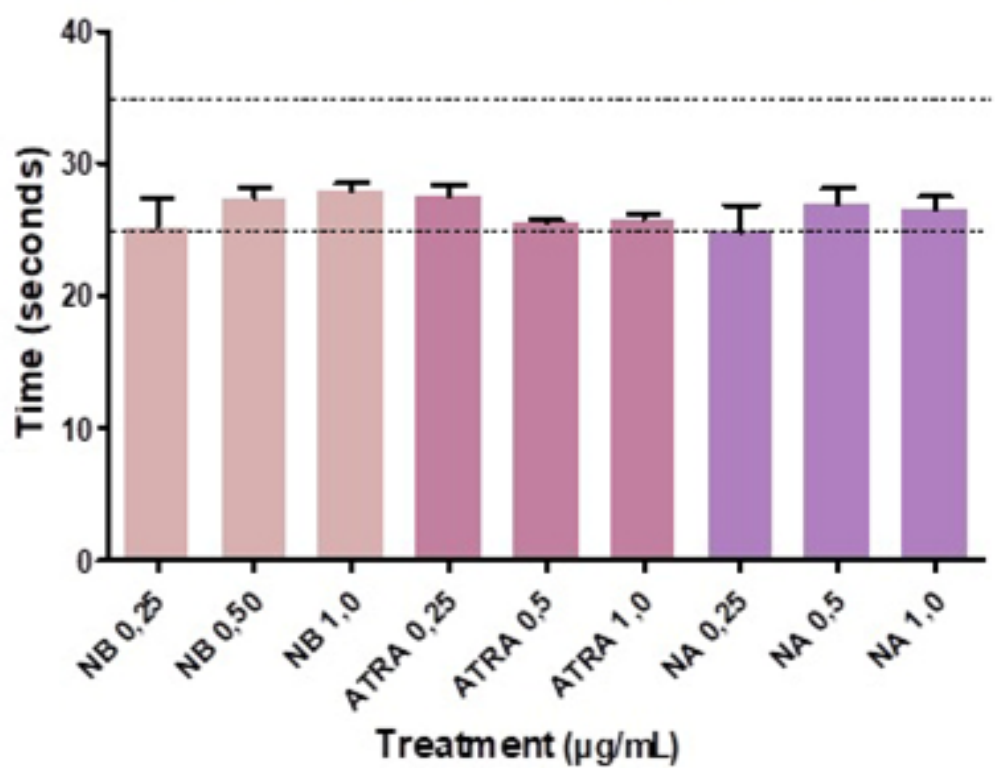

Figure 3 - APTT results

Notes: Dotted borders correspond to expected physiological times for healthy donors (between 25 and 35 seconds). Mean \pm S.D. values from two independent experiments and from different donors.

\section{DISCUSSION}

Nanomedicine has evolved in the last years with the increase of the studies in this area. Nanoparticles increasingly play an important role in drug delivery, therapy and diagnosis. Because they have unique properties such as reduced size, large surface area and high reactivity. These characteristics make these nanoparticles have different behaviors according to the interaction medium.

For a particle to be considered nanometric, it is necessary that at least one of its dimensions is smaller than 1000 nanometers and that this causes changes in its natural properties. Thus, the results found for the sizes of the nanocapsules produced agree with the criteria established in the literature. ${ }^{11}$

The PDI measures the particle size distribution, which must be homogeneously distributed ${ }^{12}$ suggests that in the case of manometric particles, the formulations should be monodispersed, in other words, with PDI values of less than about 0.25 . 
Nanoparticles may have longer systemic circulation times. These prolonged circulation time in the bloodstream increase the duration of contact with these blood components, including the coagulation system, potentially amplifying the activation of the coagulation cascade. ${ }^{13}$

In APL, malignant promyelocytes reveal procoagulant substances that activate the coagulation cascade, usually thrombin, release fibrinogen, coagulation factors, and platelets. Because of this reaction, patients with APL show DIC, fibrinolysis and proteolysis. ${ }^{14}$

The fibrinolytic system is generally largely inactivated during the phase of increased activation of coagulation, which contributes to the deposition of fibrin. However, in some situations (for example, in APL) fibrinolysis may be accelerated, thus contributing to severe bleeding. ${ }^{15}$

Fibrin deposition may lead to occlusion of the vessels and consequent impairment of blood supply to various organs, which, together with metabolic and hemodynamic changes, contribute to the failure of multiple organs. Consumption and consequent depletion of coagulation factors and platelets resulting from continuous procoagulant activity may lead to diffuse bleeding, which is often the first manifestation noted. It is important to emphasize that the DIC is always secondary to a basic disease and the identification and treatment of the predisposing condition are fundamental for the resolution of the syndrome. ${ }^{16}$

Morbidity and mortality in APL are strongly related to coagulopathy.17 Clinically, APL differs from other Acute Myeloid Leukemias (AML) because it is associated with this alteration in about $60 \%$ to $90 \%$ of patients, the main cause of high mortality rates.12 Hemostasis alterations were studied in 20 patients with APL prior to treatment, during induction of remission and after complete remission. The DIC was detected in 14 cases, $71 \%$ of them at the time of diagnosis. ${ }^{17}$

The results found in the hemolytic activity of free ATRA, evidenced the increase of the same, dependent concentration, corroborating with the findings Lim, Lee and Kim.18 ATRA in free form has poor solubility, is chemically unstable, and when exposed to different factors, such as light, heat and oxidants, rapidly turns into isomerized products, such as isotretinoin or oxidized products such as all-trans-4-oxo. ATRA is highly lipophilic and has 
this ability to permeate phospholipid bilayers, resulting in destabilization of erythrocyte cell membranes. ${ }^{19}$

With these findings and reporting to the clinic of patients with APL who acquire the syndrome of Differentiation to the ATRA, they are found to have symptoms of medullary relapse and severe anemia due to the use of high doses of this chemotherapy by the vein. ${ }^{19}$

Because ATRA is a hydrophobic drug, it has low aqueous solubility, it is a major disadvantage to administer it intravenously. But numerous efforts have been made to allow its administration by this route using nanocarriers, especially lipid systems such as liposomes, nanoemulsions (NE) and solid lipid nanoparticles (SLN) as a drug delivery system. ${ }^{18}$

No study was found, although the activity of the nanoparticles loaded with ATRA had the development team, yet they induced lower haemolytic toxicity. Moreover, these systems present potential for accumulation of the drug through a passive targeting called permeability and increased retention (EPR). ${ }^{20}$

Which is a complex process, activated by fibrinogen and involving other proteins. It is divided into two pathways: the intrinsic (antibody-dependent) and extrinsic (nonantibody-dependent) pathway, both ending in a common pathway.

However, the trends observed in studies of polymer-based nanoparticles, similar to that studied by us, are related to charge dependence as well as to hemolytic activity. Koziara, Oh, Akers, Ferraris, and Mumper13 showed that the reduction of the particle surface charge decreases platelet aggregation and activation. Previous studies have shown that polymeric nanoparticles injected intravenously could modify coagulation time by both pathways. ${ }^{13}$

Thus, the extrinsic coagulation pathway was measured using the PT test followed by the evaluation of the intrinsic coagulation pathway with the APTT. As previously mentioned, all treatments showed anticoagulants for concentrations higher than $1.5 \mu \mathrm{g} /$ $\mathrm{mL}$ for the TTPA test, in the TP test the ATRA treatment maintained the results standard as the previous test, but in the nanoparticle treatments the anticoagulant activity was observed from the concentration of $1.0 \mu \mathrm{g} / \mathrm{mL}$. As the main alterations occurred in the PT test, it is believed that a greater interaction with the factors VII, X, V, II of coagulation and fibrinogen could have occurred, however, as alterations were observed in the second test 
(APTT), we did not rule out interactions with the intrinsic factors of coagulation, among them, factor VIII, IX, XI, XII and protein Precalicrine. ${ }^{21}$ Other methods of analysis should be performed to confirm and better understand the results obtained here.

\section{CONCLUSION}

In conclusion of the interaction of the nanoparticles with the hemolysis tests and the coagulation cascade, we observed the occurrence of hemolysis with the nanoparticles with ATRA, but with a decrease in its toxicity compared to the free ATRA. The results for coagulation cascade showed that the two lowest concentrations present a safe profile, without intrinsic and extrinsic pathways alteration. Thus, we believe that there is a promising benefit to the use of these developed nanoparticles, but in vitro and in vivo cytogenotoxicity assays are necessary to ensure the use of these nanocarreadores aiming pharmacological safety of them.

\section{ACKNOWLEDGEMENTS}

This work was performed with financial support from research agency: FAPERGS EDITAL 01/2017- ARD (17/2551-0000805-7).

\section{REFERENCES}

1. Craig SK, Barbara K, Craig MK. Consultative hemostasis and thrombosis. 2nd ed. Rio de Janeiro: Saunders; 2007.

2. Levi M, Ten CH. Disseminated intravascular coagulation. N Engl J Med. 1999; 341:58692.

3. Asou N. All-trans retinoic acid in the treatment of acute promyelocytic leukemia. Intern Med. 2007; 46(2):91-3.

4. Marshall JC. Inflammation, coagulopathy, and the pathogenesis of multiple organ dysfunction syndrome. Crit CareMed. 2001; 29:S99-106. 
5. Fachinetto JM, Ourique AF, Lubini G, Tedesco SB, Silva ACF, Beck RCR. Tretinoin-loaded polymeric nanocapsules: evaluation of the potential to improve the antiproliferative activities on Allium ceparoot-tip compared to the free drug. Lat Am J Pharm. 2008; 27(5):668-73.

6. Ourique AF, Azoubel S, Ferreira CV, Silva CB, Marchiori MCL, Pohlmann AR, et al. Lipid-core nanocapsules as a nanomedicine for parenteral administration of tretinoin: development and in vitro antitumor activity on human myeloid leukaemia cells. J. Biomed. Nanotechnol. 2010; 6:214-23.

7. Fessi H, Puisieux F, Devissaguet JPh. Ammoury N, Benita S. Nanocapsule formation by interfacial Polymer deposition following solvent displacement. Internacional J pharm; 1989:R1-R4.

8. Dobrovolskaia MA, Patri AK, Zheng J, Clogston JD, Ayub N, Aggarwal P, et al. Interaction of colloidal gold nanoparticles with human blood: effects on particle size and analysis of plasma protein binding profiles. Nanomedicine; 2009; 5:106-17.

9. Salvador-Morales C, Zhang L, Langer R, Farokhzad OC. Immunocompatibility properties of lipid-polymer hybrid nanoparticles with heterogeneous surface functional groups. Biomaterials; 2009; 30:2231-40.

10. Adamson PC, Pitot HC, Balis FM, Rubin J, Murphy RF, Poplack DG. Variability in the oral bioavailability of all-trans-retinoic acid. J Natl Cancer Inst; 1993; 85(12):993-6.

11. Auffan M, Rose J, Bottero JY, Lowry GV, Jolivet JP, Wiesner MR. Toward a definition of inorganic nanoparticles from an enviromental, health and safety perspective. Nat Nanotechnol. 2009; 4.

12. Jácomo RH, Figueiredo-Pontes LL, Rego EM. From the molecular model to the impact on prognosis: an overview on acute promyelocytic leukimia. Rev. Assoc. Med. Bras. 2008; 54(1):82-9.

13. Koziara JM, Oh JJ, Akers WS, Ferraris SP, Mumper RJ. Blood compatibility of cetyl alcohol/polysorbate-based nanoparticles. Pharm. Res. 2005; 22(11): 1821-28.

14. Tallman MS. Therapy of acute promyelocytic leukemia: all-trans retinoic acid and beyond. Leukemia; 1999; 12:37-40.

15. Ten $\mathrm{CH}$, Timmerman JJ, Levi M. The pathophysiology of disseminated intravascular coagulation. Thromb Haemost. 1999; 82:713-7. 
16. Taylor FB Jr, Toh CH, Hoots WK, Wada H, Levi M. Towards definition, clinical and laboratory criteria, and a scoring system for disseminated intravascular coagulation. Thromb Haemost. 2001; 86:1327-30.

17. Choudhry A, Deloughery TG. Bleeding and thrombosis in acute promyelocytic leukemia. Am J hematol. 2012; 87(6):596-603.

18. Lim SJ, Lee MK, Kim CK. Altered chemical and biological activities of all-trans retinoic acid incorporated in solid lipid nanoparticle powders. J Control Release. 2004; 100(1):53-61.

19. Hogan CJ, Wiley JS, Billington T. Intravascular haemolysis complicating treatment of acute promyelocytic leukaemia wih all-trans retinoic acid(ATRA). Aust. N.Z.J. Med. 1997; 27:450-1.

20. Sultana S, Khan MR, Kumar M, Kumar S, Ali M. Nanoparticles-mediated drug delivery approaches for cancer targeting: a review. J. Drug Target. 2012.

21. Hunt RL. Evaluation of the prothrombin time, a PTT, and platelet count in the bleeding infant. Neonatal Netw. 2011; 30:253-6.

Recebido em: 22 de março de 2018 Avaliado em: 09 de outubro de 2018 (Avaliador A) Avaliado em: 31 de julho de 2018 (Avaliador B) Aceito em: 10 de outubro de 2018 